\title{
Aberration and the Question of Equivalence of some Ether Theories to Special Relativity
}

\author{
François Goy \\ Dipartimento di Fisica, Università di Bari \\ Via G. Amendola 173 \\ I-70126 Bari, Italy \\ E-mail: GOY@AXPBA1.BA.INFN.IT
}

December 7,95

\begin{abstract}
In the last two decades, theories explaining the same experiments as well as special relativity does, were developed by using different synchronization procedures. All of them are ether-like theories. Most authors believe these theories to be equivalent to special relativity, but no general proof was ever brought. By means of a Gedankenexperiment on light aberration, we produce strong evidence that this is the case for experiments made in inertial systems.
\end{abstract}

Keywords: special relativity, ether, velocity of light, stellar aberration, synchronization.

\section{Introduction}

In his famous article of 1905, Einstein[1] gave a definition of time in a point B, in terms of the time of a distant point $\mathrm{A}$ in the same inertial system, by postulating that the "time" which light needs to go from A to B is the same as the "time" needed to go from B to A. He then showed that this synchronization procedure is consistent with his relativity principle.

More recently, Mansouri and Sexl[2] have constructed a set of transformations between inertial systems, based on other synchronizations, which depend on a parameter $\varepsilon$. They showed that only the value of $\varepsilon$ given by the Einstein's synchronization procedure is compatible with the principle of relativity and gives a one-way velocity of light constant in all inertial frames. In particular, the choice of the so-called absolute synchronization leads to an ether-like theory (Theory of Inertial Transformations(TIT)) 
which has transformations different from the Lorentz-ones, maintains absolute simultaneity, and has a one-way velocity of light different from c. It was claimed by the authors, that this ether-like theory is kinematically equivalent to the Special Relativity Theory(SRT). This implies that it is impossible to measure the velocity of an inertial frame relative to the ether, or equivalently, that the one-way velocity of light is a convention, or that the synchronization procedure is a matter of choice. This is sometimes called Poincaré principle, but it is a conjecture that was really never proved.

Sjödin [3] developed waves and Maxwell equations for all synchronization procedures. He stated that we can only measure the "absolute" velocity of inertial systems by means of tachyons or waves travelling through the ether at a velocity different from c. Since such things have never been observed and probably do not exist in nature, this idea has no practical consequences.

Selleri [4] developed the dynamical part of the TIT and the general transformations between inertial systems. He gave an argument based on the Sagnac effect claiming the logical inequivalence of the SRT and TIT. In fact, if there were only one different prediction between the TIT and the SRT, it would imply that the one-way velocity of light is measurable independently of conventions.

Aberration of light is a phenomenon in which only the one-way velocity of light come into play and in which, apparently, no clocks are used. Thus we think it is a good test for the equivalence of the SRT and the TIT. Sjödin and Podlaha [5] wrote an article on the subject but their earth was at rest always in the same inertial frame, thus an idealized solar system very different from the real one.

We develop here a Gedankenexperiment using aberration of light with two different inertial frames and conclude that the SRT leads to the same results as the TIT.

\section{Synchronization and measurement of velocities}

Let $K$ be an inertial frame having velocity $\boldsymbol{v}$ relative to the fundamental frame $K_{0}$ along the $x_{0}$-axis in positive direction. Along the $x$-axis of $K$, there are two points $\mathrm{A}$ and $\mathrm{B}$ with $x_{\mathrm{A}}<x_{\mathrm{B}}$ and $\left|\boldsymbol{x}_{\mathrm{B}}-\boldsymbol{x}_{\mathrm{A}}\right|=d$. In A there is one clock and in $\mathrm{B}$ there are two clocks.

We synchronize the first clock in B with the one in A by using Einstein's procedure. It means that a light ray is sent from $\mathrm{A}$ at time $t_{\mathrm{A}}$ reflected in $\mathrm{B}$ at time $\tilde{t}_{\mathrm{B}}$ (where stands for Einstein's synchronization) and comes back to A at time $t_{\mathrm{A}}^{*}$. The definition of $\tilde{t}_{\mathrm{B}}$ is

$$
\tilde{t}_{\mathrm{B}}=\frac{t_{\mathrm{A}}^{*}+t_{\mathrm{A}}}{2}
$$

One can easily verify that this definition is based on the assumption that the velocity of light is the same from A to B as from B to A. If an object on which no forces act leaves $\mathrm{A}$ at time $t_{1}$ and reaches $\mathrm{B}$ at time $\tilde{t}_{2}$, its velocity $\tilde{v}_{1}$ is

$$
\tilde{v}_{1}=\frac{d}{\tilde{t}_{2}-t_{1}}
$$


Then, we synchronize the second clock in B with a procedure of "absolute" synchronization, which takes account of the fact that the velocity of light $c_{\mathrm{AB}}$ from $\mathrm{A}$ to $\mathrm{B}$ is different from the velocity $c_{\mathrm{BA}}$ from $\mathrm{B}$ to $\mathrm{A}$. The TIT gives (see Ref. [4])

$$
c_{\mathrm{AB}}=\frac{c}{1+\beta} ; \quad c_{\mathrm{BA}}=\frac{c}{1-\beta}
$$

where $c$ is the two-way velocity of light and $\beta$ stands for $\frac{v}{c}$. A light ray is sent from $\mathrm{A}$ at time $t_{\mathrm{A}}$, reflected in $\mathrm{B}$ at time $t_{\mathrm{B}}$ and comes back to $\mathrm{A}$ at time $t_{\mathrm{A}}^{*}$. Using (3), we have:

$$
d=c_{\mathrm{AB}}\left(t_{\mathrm{B}}-t_{\mathrm{A}}\right)=\frac{c\left(t_{\mathrm{B}}-t_{\mathrm{A}}\right)}{1+\beta}=c_{\mathrm{BA}}\left(t_{\mathrm{A}}^{*}-t_{\mathrm{B}}\right)=\frac{c\left(t_{\mathrm{A}}^{*}-t_{\mathrm{B}}\right)}{1-\beta}
$$

Comparing the third and the fifth term of this equality, we obtain:

$$
t_{\mathrm{B}}=\frac{t_{\mathrm{A}}^{*}+t_{\mathrm{A}}}{2}+\frac{\beta\left(t_{\mathrm{A}}^{*}-t_{\mathrm{A}}\right)}{2}
$$

In both theories, the two-way velocity of light is constant in all directions so that:

$$
t_{\mathrm{A}}^{*}-t_{\mathrm{A}}=\frac{2 d}{c}
$$

Using (11) and (6), Eq. (5) becomes:

$$
t_{\mathrm{B}}=\tilde{t}_{\mathrm{B}}+\frac{\beta d}{c}
$$

The velocity $v_{1}$ of the same object as before is measured. It leaves $\mathrm{A}$ at time $t_{1}$ and reaches $\mathrm{B}$ at time $t_{2}$. Using (2) and (7) with $t_{\mathrm{B}}=t_{2}$ and $t_{\mathrm{A}}=t_{1}$, we obtain:

$$
v_{1}=\frac{d}{t_{2}-t_{1}}=\frac{d}{\tilde{t}_{2}-t_{1}+\frac{\beta d}{c}}=\frac{1}{\frac{1}{\tilde{v}_{1}}+\frac{\beta}{c}}=\frac{\tilde{v}_{1}}{1+\beta \tilde{\beta}_{1}}
$$

where $\tilde{\beta}_{1}$ stands for $\frac{\tilde{v}_{1}}{c}$. In the same physical situation, an observer will not obtain the same numerical value of the velocity if he uses different synchronization procedures, but (8) brings a connection between them. Note that the results obtained here for the time $t_{\mathrm{B}}$ or the velocity $v_{1}$ can easily be generalized to the case of a vector $\overrightarrow{A B}$ making an angle $\psi$ with $\boldsymbol{v}$ : at every place where $\beta$ appears it has to be replaced by $\beta \cos \psi$.

\section{Aberration}

Stellar aberration, discovered by Bradley in 1728, is an apparent motion of all stars during the year along an ellipse, whose major axis approaches 41" and is explained by the classical formula

$$
\Delta \theta=\frac{\tilde{v}_{1}}{c} \sin \theta \quad \text { for } \quad \frac{\tilde{v}_{1}}{c} \ll 1
$$


where $\theta$ is the angle between the earth velocity and the light ray. $\Delta \theta$ is the difference between the angles that light makes in two reference frames moving at relative velocity $\tilde{v}_{1}$ (here the earth at different times of the year), and $c$ is the two-way speed of light.

In the SRT, the aberration is only a matter of motion of the observer's inertial frame relative to another inertial frame (see for example Ref. [6]). Considering an inertial system $K$ and another $K^{\prime}$ moving with velocity $\tilde{\boldsymbol{v}}_{\mathbf{1}}$ relative to $K$ along the $x$-axis in positive direction, we have:

$$
\tan \tilde{\theta}^{\prime}=R\left(\tilde{\beta}_{1}\right) \frac{\sin \theta}{\cos \theta+\tilde{\beta}_{1}}
$$

where $\theta+\pi$ is the angle between the light velocity vector and the $x$-axis,$\tilde{\theta}^{\prime}+\pi$ is the same quantity in $K^{\prime}$ and $R\left(\tilde{\beta}_{1}\right)$ stands for $\sqrt{1-\tilde{\beta}_{1}^{2}}$. There is no tilda on $\theta$ because this angle does not depend at all on synchronisation, but there is one on $\tilde{\theta}^{\prime}$ because this angle is a function of $\tilde{v}_{1}$ in Eq. (10).

Note that (9) was derived from a classical equation similar to (10) but without the relativistic factor $R\left(\tilde{\beta}_{1}\right)$. Within the actual precision of measurement, this factor is not observable because the next term of $\Delta \theta$ is of order $\left(\frac{\tilde{v}_{1}}{c}\right)^{3}$ when $\theta=\pi / 2$ and (10) reduces to (9) for $\tilde{v}_{1} \ll c$.

Many textbooks and the original Einstein article explain aberration from the relative motion of an observer and the source. It has been shown that this explanation cannot be correct, because otherwise binary stars should present an aberration larger than other stars and they do not (see Refs. [7, 8, 9, 10]). The right relativistic explanation is the one given above.

In the TIT, the aberration equation depends on the "absolute" velocity of the observer. Let $K$ be an inertial frame moving with velocity $\boldsymbol{v}$ relative to the privileged frame $K_{0}$ along the $x_{0}$-axis in positive direction and another inertial frame $K^{\prime}$ moving with velocity $\boldsymbol{v}^{\prime}$ along the $x_{0}$-axis in the positive direction. The inertial transformations in $2+1$ dimensions between $K$ and $K^{\prime}$ (see Refs. [4]) are, with an obvious notation:

$$
\begin{aligned}
x^{\prime} & =\frac{R(\beta)}{R\left(\beta^{\prime}\right)}\left[x-\frac{\left(\beta^{\prime}-\beta\right) c}{R^{2}(\beta)} t\right] \\
y^{\prime} & =y \\
t^{\prime} & =\frac{R\left(\beta^{\prime}\right)}{R(\beta)} t
\end{aligned}
$$

where $R(\beta)=\sqrt{1-\beta^{2}}$ and $R\left(\beta^{\prime}\right)=\sqrt{1-\beta^{\prime 2}}$. Writing (111) in differential form and dividing the space variables by the time variable, we obtain the transformations of velocity components:

$$
\begin{aligned}
& u_{x}^{\prime}=\frac{R^{2}(\beta)}{R^{2}\left(\beta^{\prime}\right)}\left[u_{x}-\frac{\left(\beta^{\prime}-\beta\right) c}{R^{2}(\beta)}\right] \\
& u_{y}^{\prime}=\frac{R(\beta)}{R\left(\beta^{\prime}\right)} u_{y}
\end{aligned}
$$


where $\boldsymbol{u}$ and $\boldsymbol{u}^{\prime}$ are the velocities in $K$ and $K^{\prime}$, respectively. We refer now to the propagation of a light pulse whose velocity $\boldsymbol{c}_{\mathrm{K}}$ in $K$ makes an angle $\theta+\pi$ with the $x$-axis (resp. $\boldsymbol{c}_{\mathrm{K}^{\prime}}$ and $\theta^{\prime}+\pi$ in $K^{\prime}$ ). We have by projection on the $x-, y-, x^{\prime}-$, and $y^{\prime}$-axis, respectively:

$$
u_{x}=-c_{\mathrm{K}} \cos \theta ; \quad u_{y}=-c_{\mathrm{K}} \sin \theta ; \quad u_{x}^{\prime}=-c_{\mathrm{K}^{\prime}} \cos \theta^{\prime} ; \quad u_{y}^{\prime}=-c_{\mathrm{K}^{\prime}} \sin \theta^{\prime}
$$

Replacing (13) in (12) and dividing side by side leads to the aberration formula:

$$
\tan \theta^{\prime}=\frac{R\left(\beta^{\prime}\right)}{R(\beta)} \frac{\sin \theta}{\cos \theta+\frac{\left(\beta^{\prime}-\beta\right)(1-\beta \cos \theta)}{R^{2}(\beta)}}
$$

where we have used the fact that in this case $c_{\mathrm{K}}=\frac{c}{1-\beta \cos \theta}$. When $\beta=0$, (14) reduces to an equation similar to (10)

$$
\tan \theta^{\prime}=R\left(\beta^{\prime}\right) \frac{\sin \theta}{\cos \theta+\beta^{\prime}}
$$

but there is a great conceptual difference between (10) and (15) since in (15), $\beta^{\prime} c$ is a velocity relative to the priviledged frame and in (10), $\tilde{\beta}_{1} c$ is the velocity between two arbitrarly choosen inertial frames.

The physical basis of aberration is the fact that the velocity of light is finite and changes its direction when seen from another reference frame. It is a consequence of the velocity addition formula applied to a light ray when the observer is changing its reference frame. There is also an "aberration effect" at emission. When the source emits in all directions it cannot be observed, but when the source emits in only one direction, a non-uniform motion of the source clearly changes the direction of emission, since the velocity addition formula can also be applied. Corrections for the "aberration" of the source are used in the determination of the true pulsar period in binary systems[1].

Note that in both theories, only the difference $\Delta \theta$ is observable.

\section{Aberration and delay effect}

From (15) one could think that in ether-theories the aberration $\theta^{\prime}-\theta$ due to an uniform motion relative to $K_{0}$ should be observable. In the SRT such a problem does not occur since there is no privileged frame. Prokhovnik and Morris [9] wrote: "Certainly, an observer travelling at very great speed (say c/2) relative to the universe would have a very distorded and asymmetric view of the distribution of the stars and galaxies as a result of the aberration effect." In the same article, they explained that, for nearby sources the aberration is cancelled by a delay effect. In this section, we show that the cancellation of aberration by a delay effect as nothing to do with the nearness of the source but only with the uniformity of the motion of the observer, so that an observer travelling at very great uniform speed would see no distortion of the distribution of the stars. On the contrary, an observer in a laboratory on earth would 


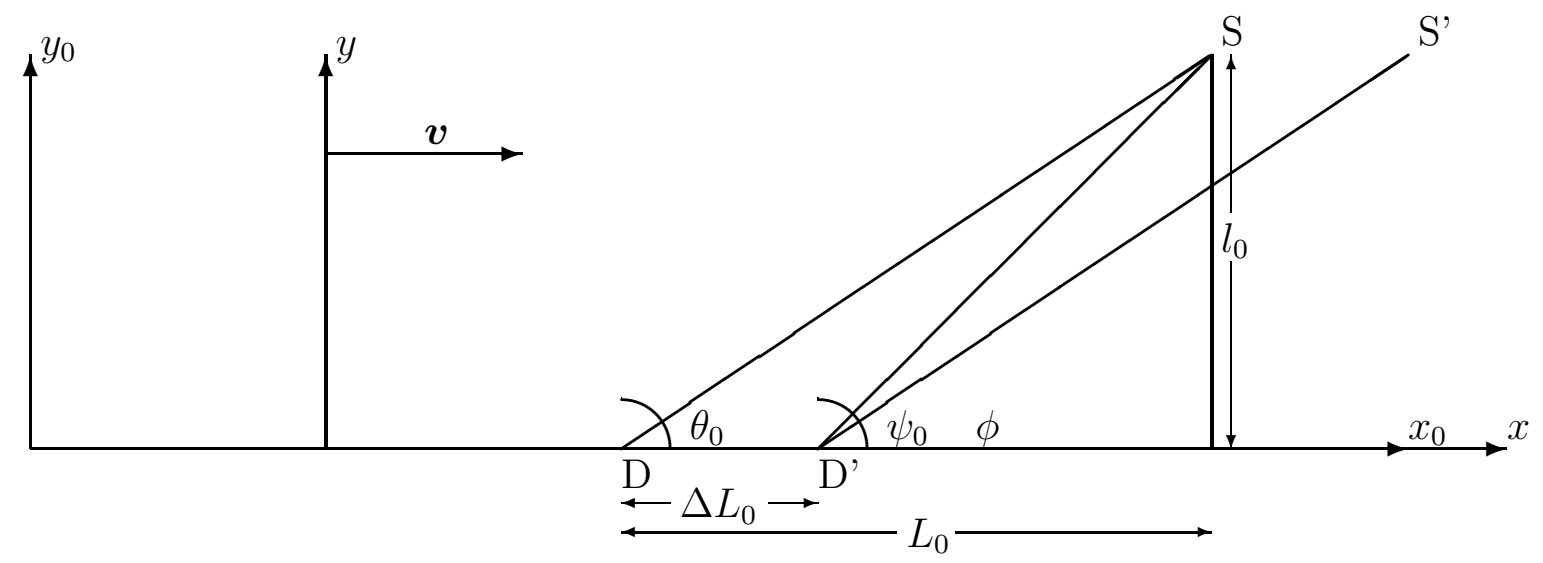

Figure 1: The source $\mathrm{S}$ begins to emit at $t_{0}=0$ but the detector in $\mathrm{D}$ at $t_{0}=0$ recieves the signal only later in D' and with an angle $\psi_{0}$ (in $K_{0}$ ). Since the detector is at rest in $K, \psi_{0}$ will be aberrated and seen as $\phi$ in $K$. An observer in $K$ finds that $\phi=\theta$

see the beam of a laser moving of 41 " in six month since the motion of the earth is not uniform.

Let us imagine the following situation: a detector D and a source $\mathrm{S}$ emitting in all directions are at rest in the reference frame $K$ of section 3. For an observer in $K_{0}$, the following quantities are defined (see figure 1): $l_{0}$ is the distance from $\mathrm{S}$ to the $x_{0}$-axis, $L_{0}$ is the projection of DS on the $x_{0}$-axis, $\tan \theta_{0}=\frac{l_{0}}{L_{0}}$. $\theta_{0}$ is known by measuring $L_{0}$ and $l_{0}$. One could think that by measuring the $\theta^{\prime}$ of (15), it is possible to determine the velocity of $K$ relative to $K_{0}$. It is not so because of a delay effect. At time $t_{0}=0$ of $K_{0}$ the source $\mathrm{S}$ begin to emit. The detector $\mathrm{D}$ will have moved a distance $\Delta L_{0}$ before light from $\mathrm{S}$ reaches it. The time $t_{0}$ of reception is

$$
t_{0}=\frac{\sqrt{l_{0}^{2}+\left(L_{0}-\Delta L_{0}\right)^{2}}}{c}=\frac{\Delta L_{0}}{v}
$$

so that,

$$
\Delta L_{0}=\frac{\beta}{R^{2}(\beta)}\left(-L_{0} \beta+\sqrt{\left(R(\beta) l_{0}\right)^{2}+L_{0}^{2}}\right)
$$

The angle $\psi_{0}$ due to a delay effect is the angle at which light falls on $\mathrm{D}$ in $K_{0}$ and can now be calculated as:

$$
\tan \psi_{0}=\frac{l_{0}}{L_{0}\left(1-\frac{\Delta L_{0}}{L_{0}}\right)}=\frac{\sin \theta}{R(\beta)\left[\cos \theta-\frac{\beta(1-\beta \cos \theta)}{R^{2}(\beta)}\right]}
$$

where we have used $\theta$, the angle in $K$ corresponding to $\theta_{0}$ given by the transformations:

$$
L=\frac{L_{0}}{R(\beta)} ; \quad \Delta L=\frac{\Delta L_{0}}{R(\beta)} ; \quad l=l_{0} ; \quad \tan \theta=R(\beta) \tan \theta_{0} .
$$

The angle $\psi_{0}$ is seen as $\phi$ in $K$. We have the following aberration relation.

$$
\tan \psi_{0}=\frac{\sin \phi}{R(\beta)\left[\cos \phi-\frac{\beta(1-\beta \cos \phi)}{R^{2}(\beta)}\right]}
$$


The relation (20) is given here for the need of the proof in its inverse form and was obtained by putting $\beta^{\prime}=0, \psi_{0}$ in place of $\theta^{\prime}$ and $\phi$ in place of $\theta$ in (14). Let us write relation (18) as $\psi_{0}=\mathcal{F}(\theta)$ and relation (20) as $\psi_{0}=\mathcal{G}^{-1}(\phi)$ so that we obtain:

$$
\phi=\mathcal{G}\left(\psi_{0}\right)=\mathcal{G}(\mathcal{F}(\theta))=\theta
$$

since $\mathcal{F}=\mathcal{G}^{-1}$. It means that an observer in $K$ measures an angle $\phi$ exactly equal to $\theta=\arctan \left(\frac{l}{L}\right)$. So in the TIT, the aberration due to a uniform motion is not observable. No assumptions was made concerning $l_{0}$ and $L_{0}$, so that the result obtained here is exactly the same if the source is near to or far from the detector solong the motion is uniform.

\section{Gedankenexperiment}

Aberration of light is typically a physical phenomenon in which the one-way velocity of light comes into play. Sjödin and Podlaha[5] wrote about stellar aberration: "The only cause of the effect is the change of the relative velocity of the earth during the year.(...). The use of so-called absolute synchronisation could, therefore, impossibily have an influence on the observed effect." The point is that Sjödin and Podlaha used in their proof only one inertial frame representing the earth, thus a frame which is totally unable to represent the change of the velocity of the earth during the year. As a better approximation to reality we propose here a Gedankenexperiment with two different reference frames.

Let us begin with the SRT. A spaceship is at rest in an inertial system $K$. On the inside, one prepares a laser so that its beam makes an angle $\theta$ with the wall of the rocket which is also the future direction of acceleration. Then the rocket accelerates and reaches a velocity $\tilde{\boldsymbol{v}}_{\mathbf{1}}$ relative to $K$ (see figure 2). Of course in $K$, clocks are synchronized with Einstein's procedure, and $\tilde{v}_{1}$ is measured with such clocks. The angle $\tilde{\theta}^{\prime}$ after acceleration is given by (10).

Let us now consider the same experiment in the TIT, but imagine that $K$ was already moving with a velocity $\boldsymbol{v}$ relative to $K_{0}$. For simplicity, we suppose that the rocket reaches a velocity $\boldsymbol{v}_{1}$ relative to $K$ which is parallel to $\boldsymbol{v}$ and has the velocity $\boldsymbol{v}^{\prime}$ relative to $K_{0}$. In the TIT, clocks are synchronized with an "absolute" procedure and $v_{1}$ is measured with these clocks. $v^{\prime}$ is given by the first part of (12), with $u_{x}^{\prime}=0$ and $u_{x}=v_{1}$, respectively . Since we want to predict aberration in the same physical situation as above, and also be able to compare the results with those of the SRT, we express $v_{1}$ in terms of $\tilde{v}_{1}$ and $v$ by means of (8). We obtain:

$$
v^{\prime}=v+\frac{\tilde{v}_{1}}{1+\beta \tilde{\beta}_{1}} R^{2}(\beta)
$$

The angle $\theta^{\prime}$ is now given by (14) which seems to be very different from (10) and in particular to depend on $v$. It is in fact not the case. Replacing $v^{\prime}$ in (14) by its value (22), one obtains after a few calculations that (14) is exactly the same as (10). So there is no observable difference between the SRT and the TIT in this case. 


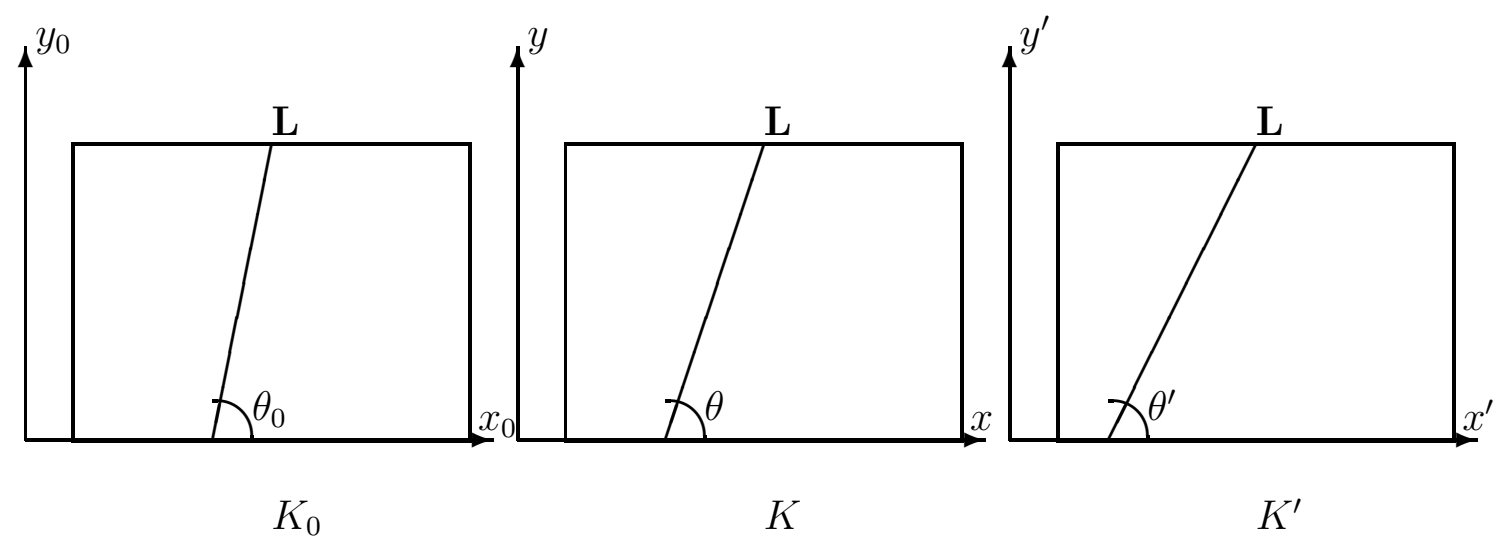

Figure 2: Different angles laser beam/x-axis. 1 . In the TIT: $K_{0}$ (privileged frame), $K$ (initial inertial frame; "absolute" velocity: $\boldsymbol{v}$ ), and $K^{\prime}$ (inertial frame after acceleration; "absolute" velocity: $\boldsymbol{v}^{\prime}$; velocity relative to $\left.K: \boldsymbol{v}_{\mathbf{1}}\right)$. 2. In the SRT: $K$ (initial inertial frame), $K^{\prime}$ (inertial frame after acceleration; velocity relative to $K$ : $\tilde{\boldsymbol{v}}_{\mathbf{1}}$. L stands for laser source.

\section{Discussion}

1. The arguments of sections 2,4 , and 5 bring strong evidences that also in other cases where one could try to obtain a difference between the TIT and the SRT by comparing an observed effect in two different inertial frames, one would also obtain the same conclusion as here.

2. Nevertheless, the conclusion we obtain here cannot be generalised further without proof. We cannot conclude that there is a general equivalence between the SRT and the TIT.

3. It is still an open question to know if the one way velocity of light is a purely conventional quantity or is fixed by nature itself. For the time being it seems that the TIT and the SRT are equivalent for all observational purpose but there is a great philosophical difference between them.

\section{Conclusion}

By means of a Gedankenexperiment, we have proved that the SRT and the TIT lead to the same results in the case of aberration of light. In spite of the fact that hundreds of experiments trying to detect an ether drift have obtained a negative result no general proof of the equivalence of theories built on different synchronizations was ever brought. The question of the conventionality of the one-way velocity of light is still open. 


\section{Acknowledgement}

I want to thank Prof. Franco Selleri for his suggestions and criticisms and Gino Lepore for his precious help.

\section{References}

[1] A. Einstein, Ann. Physik, 17, pp. 891-921, 1905.

[2] R. Mansouri and R.U. Sexl, Gen. Relat. Grav., 8, pp. 497-513, 1977; 8, pp. 515-524, 1977; 8, pp.809-813, 1977.

[3] T. Sjödin, Nuovo Cim., 51B, pp. 229-246, 1979.

[4] F. Selleri, Space, Time and Motion, special issue of the Chin. J. Syst. Eng. Electr., to be published; Found. Phys. Lett., to be published, last issue 1995; Found. Phys., to be published, first issue 1996.

[5] T. Sjödin and M.F. Podlaha, Lett. Nuovo Cim., 31, pp. 433-436, 1981.

[6] M. Harwith, Astrophysical Concepts, Wiley and Son, New-York, 1974, pp. 170173.

[7] E. Eisner, Am. J. Phys., 35, pp. 817-819, 1967.

[8] T.E. Phipps, Jr., Am. J. Phys., 57, pp. 549-551, 1989.

[9] S.J. Prokhovnik and W.T. Morris, Found. Phys., 19, pp. 531-539, 1989.

[10] H.C. Hayden, Gal. Electrodynamics, 4, pp. 89-92, 1993.

[11] T. Damour and J.H. Taylor, Phys. Rev. D, 45, pp. 1840-1868, 1992. 